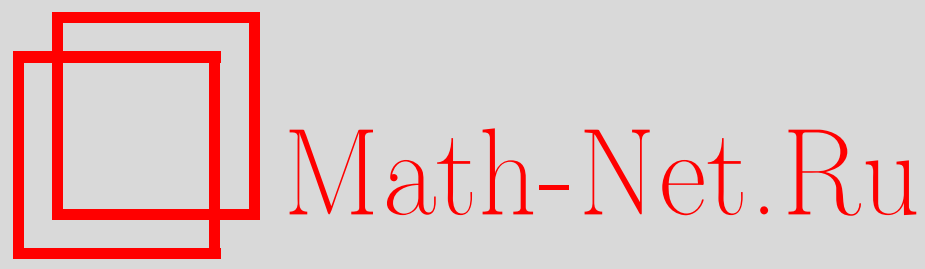

J.-Ch. Breton, Ch. Houdré, On Finite Range StableType Concentration, Теория вероятн. и ее примен., 2007, том 52, выпуск 4, 711-735

DOI: https://doi.org/10.4213/tvp1530

Использование Общероссийского математического портала MathNet.Ru подразумевает, что вы прочитали и согласны с пользовательским соглашением

http://www . mathnet.ru/rus/agreement

Параметры загрузки:

IP : 54.164 .48 .24

26 апреля 2023 г., $17: 16: 15$

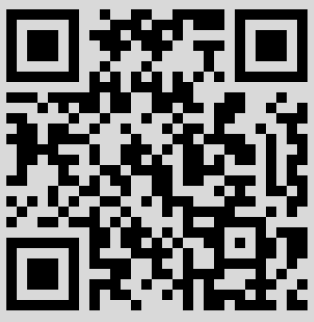


(c) $2007 \mathrm{r}$.

BRETON J.-C.*, HOUDRÉ C.**

\section{ON FINITE RANGE STABLE-TYPE CONCENTRATION ${ }^{1)}$}

Изучается вероятность отклонений $\mathbf{P}\{f(X)-\mathbf{E}[f(X)] \geqslant x\}$, где $f$ - функция, определенная на $\mathbf{R}^{d}$ и удовлетворяюшая условию Липшица (в евклидовой норме), а $X-\alpha$-устойчивый случайный вектор с индексом устойчивости $\alpha \in(1,2)$. Показывается, что эта вероятность ограничена сверху величиной $e^{-c x^{\alpha /(\alpha-1)}}$ или $e^{-c x^{\alpha}}-$ в зависимости от того, принимает $x$ малые значения или принадлежит конечному интервалу. Эти неравенства обобщаются на случай вероятности $\mathbf{P}\{F-m(F) \geqslant x\}$, где $F-$ стохастический функционал на пуассоновском пространстве с устойчивой мерой Леви индекса $\alpha \in(0,2)$ и $m(F)$ - медиана функционала $F$.

Ключевые слова и фразы: феномен концентрации меры, устойчивые случайные векторы, безграничная делимость.

\section{Introduction and preliminaries}

The purpose of these notes is to further complete our understanding of the stable concentration phenomenon, by obtaining the finite range behavior of $\mathbf{P}\{F-\mathbf{E}[F] \geqslant x\}$, with $F=f(X)$, where $f$ is a Lipschitz function and $X$ is a stable random vector, or with $F$ a stochastic functional on the Poisson space equipped with a stable Lévy measure.

More precisely, in Section 2, we consider an $\alpha$-stable random vector $X \in \mathbf{R}^{d}$ of index $\alpha \in(1,2)$ and $f: \mathbf{R}^{d} \rightarrow \mathbf{R}$ a 1-Lipschitz function and we investigate the probability of deviation from the mean, i.e.,

$$
\mathbf{P}\{f(X)-\mathbf{E}[f(X)] \geqslant x\}
$$

for all order of $x$. When $x$ takes small values, (1) is shown, in Section 2.1, to be upper bounded by $e^{-c x^{\alpha /(\alpha-1)}}$, while in Sections 2.2 and 2.3 , we give

* Laboratoire de Mathématiques et Applications, Université de La Rochelle, 17042 La Rochelle Cedex, France; e-mail: jcbreton@univ-lr.fr

** Laboratoire d'Analyse et de Mathématiques Appliqués, CNRS UMR 8050, Université Paris XII, 94010 Créteil Cedex, France; School of Mathematics, Georgia Institute of Technology, Atlanta, GA 30332, USA; e-mail: houdre@math.gatech.edu

1) The first author thanks the School of Mathematics at the Georgia Institute of Technology for the hospitality while part of this research was done. The research of the second author was supported in part by an NSA grant. 
results for intermediate values of $x$, in which case the bound is of order $e^{-c x^{\alpha}}$. In the intermediate range case, we extend the results of [4] which hold for $\alpha$ close enough to 2 , to any $\alpha \in(1,2)$. These results complement the $\left(1 / x^{\alpha}\right)$ behavior given for large values of $x$ in [3, Theorems 1,2] and [1, Theorem 6.1] and generalize [4, Theorem 2] and [1, Theorem 6.3].

In Section 3, we further extend the intermediate range results to stochastic functionals on the Poisson space $\Omega^{\mathbf{R}^{d}}$ of $\mathbf{R}^{d}$ equipped with a stable Lévy measure. In this case, the concentrations are expressed with respect to a median of the stochastic functionals and we recover as a special case the concentration of Lipschitz function of stable vector of any index $\alpha \in(0,2)$. We also briefly state the small values result for functionals when $\alpha>1$.

The main ideas of the proof are presented in Section 2. In Section 3, dealing with a median rather than with the mean makes the argument more involved, this is the reason for tackling first stable random vector of index $\alpha \in(1,2)$ in Section 2 .

The general scheme of proof is to decompose the stable random vector $X$ in two independent components $Y_{R}+Z_{R}$ with $R>0$ a level of truncation for the Lévy measure. $R$ is then chosen according to the range of concentration we are interested in. We argue similarly in Section 3 with truncated configuration $\omega_{R}=\omega \cap B(0, R), \omega \in \Omega^{\mathbf{R}^{d}}$, where $B(0, R)$ is the (Euclidean) ball of radius $R$ centered at 0 . In both cases, the remainder is controlled alternatively. We deal here only with stable Lévy measures, but more general cases (involving the function $\gamma$ present in [1]) could be tackled with similar techniques.

\section{Stable random vector of index $\alpha>1$}

In this section, we consider an $\alpha$-stable random vector $X \in \mathbf{R}^{d}$ of index $\alpha \in(1,2)$ and $f: \mathbf{R}^{d} \rightarrow \mathbf{R}$ a 1-Lipschitz function with respect to Euclidean norm $\|\cdot\|$, i.e., for all $x, y \in \mathbf{R}^{d},|f(x)-f(y)| \leqslant\|x-y\|$, and we investigate the concentration (1). The vector $X$ has an infinitely divisible law $\operatorname{ID}(b, 0, \nu)$ with Lévy measure given by

$$
\nu(B)=\int_{S^{d-1}} \sigma(d \xi) \int_{0}^{\infty} \mathbf{1}_{B}(r \xi) r^{-1-\alpha} d r, \quad B \in \mathscr{B}\left(\mathbf{R}^{d}\right),
$$

where $\sigma$ is a finite positive measure on $S^{d-1}$, the unit sphere of $\mathbf{R}^{d}$. Its characteristic function is $\varphi_{X}=e^{\psi_{X}}$ with

$$
\psi_{X}(u)=i\langle u, b\rangle+\int_{\left(\mathbf{R}^{*}\right)^{d}}\left(e^{i\langle u, y\rangle}-1-i\langle u, y\rangle \mathbf{1}_{\|y\| \leqslant 1}\right) \nu(d y),
$$

where $\left(\mathbf{R}^{*}\right)^{d}=\mathbf{R}^{d} \backslash\{0\}$. Write $X=Y_{R}+Z_{R}$, where $Y_{R}$ and $Z_{R}$ are two independent infinitely divisible random vectors with respective characteristic 
functions $\varphi_{Y_{R}}=e^{\psi_{Y_{R}}}$ and $\varphi_{Z_{R}}=e^{\psi_{Z_{R}}}$ whose exponents are given by

$$
\begin{aligned}
& \psi_{Y_{R}}(u)=i\langle u, \tilde{b}\rangle+\int_{\|y\| \leqslant R}\left(e^{i\langle u, y\rangle}-1-i\langle u, y\rangle \mathbf{1}_{\|y\| \leqslant 1}\right) \nu(d y), \\
& \psi_{Z_{R}}(u)=\int_{\|y\|>R}\left(e^{i\langle u, y\rangle}-1\right) \nu(d y),
\end{aligned}
$$

and $\tilde{b}=b-\int_{\|y\|>R} y \mathbf{1}_{\|y\| \leqslant 1} \nu(d y)$. As in [3],

$$
\begin{aligned}
& \mathbf{P}\{f(X)-\mathbf{E}[f(X)] \geqslant x\} \leqslant \mathbf{P}\left\{f\left(Y_{R}\right)-\mathbf{E}[f(X)] \geqslant x\right\}+\mathbf{P}\left\{Z_{R} \neq 0\right\} \\
& \quad \leqslant \mathbf{P}\left\{f\left(Y_{R}\right)-\mathbf{E}\left[f\left(Y_{R}\right)\right] \geqslant x-\left|\mathbf{E}[f(X)]-\mathbf{E}\left[f\left(Y_{R}\right)\right]\right|\right\}+\mathbf{P}\left\{Z_{R} \neq 0\right\} \\
& \quad \leqslant \mathbf{P}\left\{f\left(Y_{R}\right)-\mathbf{E}\left[f\left(Y_{R}\right)\right] \geqslant x-\mathbf{E}\left[\left\|Z_{R}\right\|\right]\right\}+\mathbf{P}\left\{Z_{R} \neq 0\right\},
\end{aligned}
$$

where we use the fact that $f$ is a 1-Lipschitz function. Also as in [3] (see also (15)), we know that

$$
\begin{aligned}
\mathbf{P}\left\{Z_{R} \neq 0\right\} & \leqslant 1-\exp (\nu\{\|u\| \geqslant R\})=1-\exp \left(-\int_{\|u\| \geqslant R} \nu(d u)\right) \\
& =1-\exp \left(-\int_{S^{d-1}} \sigma(d \xi) \int_{\|r \xi\| \geqslant R} \frac{d r}{r^{1+\alpha}}\right) \\
& =1-\exp \left(-\frac{\sigma\left(S^{d-1}\right)}{\alpha R^{\alpha}}\right) \\
& \leqslant \frac{\sigma\left(S^{d-1}\right)}{\alpha R^{\alpha}} .
\end{aligned}
$$

In order to study the concentration of $f\left(Y_{R}\right)$, we shall apply in Sections 2.1 and 2.2 the lemma given below. This lemma generalizes, to arbitrary order $n \geqslant 2$, Lemma 2 in [3] which corresponds to $n=2$.

Lemma 1. Let $f: \mathbf{R}^{d} \rightarrow \mathbf{R}$ be a 1-Lipschitz function, and let $Y_{R} \in \mathbf{R}^{d}$ be a random vector whose Lévy measure $\nu_{R}$ is the stable one truncated at $R>0$. Let $\delta>0$.

For any $n \geqslant 2$, let $u_{n}(\alpha, \delta)$ be a unique nonzero (thus positive) solution of

$$
e^{u}-1-\frac{\delta(n-1)}{2-\alpha} u=0
$$

and let

$$
u_{n}^{*}(\alpha, \delta)=\min _{1<k<(n-1+\alpha) / 2}\left(\left(k ! \frac{\delta(k+1-\alpha)}{(n-k)(2-\alpha)}\right)^{1 /(k-1)}\right) \wedge u_{n}(\alpha, \delta) .
$$

Then for all

$$
x \leqslant x_{0}:=(1+\delta(n-1)) \frac{\sigma\left(S^{d-1}\right) R^{1-\alpha}}{2-\alpha} u_{n}^{*}(\alpha, \delta),
$$


we have

$$
\mathbf{P}\left\{f\left(Y_{R}\right)-\mathbf{E}\left[f\left(Y_{R}\right)\right] \geqslant x\right\} \leqslant \exp \left(-\frac{(2-\alpha) x^{2}}{2 n_{\delta} \sigma\left(S^{d-1}\right) R^{2-\alpha}}\right),
$$

where $n_{\delta}=1+\delta(n-1)$.

$\mathrm{R}$ e $\mathrm{m}$ a $\mathrm{rk} 1$. In the sequel, and unless stated otherwise, $\delta$ is mainly taken to be 1 and $u_{n}(\alpha, \delta), u_{n}^{*}(\alpha, \delta)$ will simply be denoted by $u_{n}(\alpha), u_{n}^{*}(\alpha)$. We will use $\delta>0$ in Theorem 2 and Remark 4 recovering a Gaussian inequality from a stable one by letting $\delta \rightarrow 0$.

P r o of. From Theorem 1 in [2], we know that

$$
\mathbf{P}\left\{f\left(Y_{R}\right)-\mathbf{E}\left[f\left(Y_{R}\right)\right] \geqslant x\right\} \leqslant \exp \left(-\int_{0}^{x} h_{R}^{-1}(s) d s\right), \quad x>0,
$$

with the function $h_{R}$ given by

$$
h_{R}(s)=\int_{\mathbf{R}^{d}}\|u\|\left(e^{s\|u\|}-1\right) \nu_{R}(d y), \quad s>0 .
$$

Using for any $n \geqslant 2$ the inequality

$$
e^{s u}-1 \leqslant \sum_{k=1}^{n-1} \frac{s^{k}}{k !} u^{k}+\frac{e^{s K}-\sum_{k=0}^{n-1} s^{k} K^{k} / k !}{K^{n}} u^{n}, \quad 0 \leqslant u \leqslant K, \quad s \geqslant 0,
$$

we have

$$
\begin{aligned}
h_{R}(s) \leqslant & \int_{\mathbf{R}^{d}}\left(\sum_{k=1}^{n-1} \frac{s^{k}}{k !}\|u\|^{k+1}+\frac{e^{s R}-\sum_{k=0}^{n-1} s^{k} R^{k} / k !}{R^{n}}\|u\|^{n+1}\right) \nu_{R}(d y) \\
\leqslant & \sum_{k=1}^{n-1} \frac{s^{k}}{k !} \int_{B(0, R)}\|u\|^{k+1} \nu(d y) \\
& \quad+\frac{e^{s R}-\sum_{k=0}^{n-1} s^{k} R^{k} / k !}{R^{n}} \int_{B(0, R)}\|u\|^{n+1} \nu(d y) \\
\leqslant & \sum_{k=1}^{n-1}\left(\alpha_{k+1}-R^{k-n} \alpha_{n+1}\right) \frac{s^{k}}{k !}+\frac{\alpha_{n+1}}{R^{n}}\left(e^{s R}-1\right) \\
\leqslant & \sum_{k=1}^{n-1} \frac{(s R)^{k}}{k !} \frac{\sigma\left(S^{d-1}\right) R^{1-\alpha}(n-k)}{(k+1-\alpha)(n+1-\alpha)}+\frac{\sigma\left(S^{d-1}\right) R^{1-\alpha}}{n+1-\alpha}\left(e^{s R}-1\right) \\
\leqslant & \sum_{1 \leqslant k<[(n-1+\alpha) / 2]} \frac{(s R)^{k}}{k !} \frac{\sigma\left(S^{d-1}\right) R^{1-\alpha}(n-k)}{(k+1-\alpha)(n+1-\alpha)} \\
& +\left(1+n-\left[\frac{n-1+\alpha}{2}\right]\right) \frac{\sigma\left(S^{d-1}\right) R^{1-\alpha}}{n+1-\alpha}\left(e^{s R}-1\right),
\end{aligned}
$$

where we set $\alpha_{k}=\int_{B(0, R)}\|u\|^{k} \nu(d u)=\left(\sigma\left(S^{d-1}\right) /(k-\alpha)\right) R^{k-\alpha}$ and where the last line follows from the fact that for $k \geqslant(n-1+\alpha) / 2$, the $k$-th 
summand in the above sum is bounded by the last exponential term since $n-k \leqslant k+1-\alpha$.

For $1<k<(n-1+\alpha) / 2$, set

$$
v_{k}(\alpha)=\left(k ! \frac{\delta(n-1)(k+1-\alpha)}{(n-k)(2-\alpha)}\right)^{1 /(k-1)}
$$

and observe that for $s R \leqslant v_{k}(\alpha)$, the $k$-th term in the max is bounded by $\delta$ times the first one. Denote also by $u_{n}(\alpha)$ the unique positive solution of

$$
e^{u}-1-\frac{\delta(n-1)}{2-\alpha} u=0 \text {. }
$$

Next, let

$$
u_{n}^{*}(\alpha)=u_{n}(\alpha) \wedge \min _{1<k<(n-1+\alpha) / 2} v_{k}(\alpha) .
$$

For $s \leqslant R^{-1} u_{n}^{*}(\alpha)$, all the terms in (11) are bounded by $\delta$ times the linear term, so that

$$
\begin{aligned}
h_{R}(s) & \leqslant(1+\delta(n-1)) \frac{\sigma\left(S^{d-1}\right) R^{1-\alpha}}{n+1-\alpha} \frac{\delta(n-1)}{2-\alpha} s R \\
& \leqslant(1+\delta(n-1)) \frac{\sigma\left(S^{d-1}\right) R^{1-\alpha}}{2-\alpha} s R .
\end{aligned}
$$

Hence, for $t \leqslant x_{0}:=(1+\delta(n-1))\left(\sigma\left(S^{d-1}\right) R^{1-\alpha} /(2-\alpha)\right) u_{n}^{*}(\alpha)$, we can take

$$
h_{R}^{-1}(t)=\frac{(2-\alpha) t}{(1+\delta(n-1)) \sigma\left(S^{d-1}\right) R^{2-\alpha}}
$$

which finally yields (8) from (9) after easy computations. Lemma 1 is proved.

$\mathrm{R}$ e m a r k 2 (on $\left.v_{k}(\alpha), u_{n}(\alpha), u_{n}^{*}(\alpha), x_{0}(n)\right)$. From now on and unless stated otherwise, $\delta=1$.

1. The larger $n$, the worse the concentration (8) becomes.

2. For $n \leqslant 3$, the range $1<k<(n-1+\alpha) / 2$, in the minimum defining $u_{n}^{*}(\alpha)$, is empty and thus $u_{n}^{*}(\alpha)=u_{n}(\alpha)$.

3. As in (6.6) of [1], we have

$$
\ln \left(\frac{n-1}{2-\alpha}\right)<u_{n}(\alpha)<2 \ln \left(\frac{n-1}{2-\alpha}\right) .
$$

Moreover, for $n$ respectively larger than $5,13,18$, observe that we have $u_{n}(\alpha) \geqslant 1,2,3$.

4. We give more explicit expression for the domain in (7) in some concrete cases. It is easy to see that for $k \geqslant 2$,

$$
k ! \frac{n-1}{n-k} \frac{k+1-\alpha}{2-\alpha} \geqslant k ! \frac{3-\alpha}{2-\alpha} \geqslant 3^{k-1},
$$


so that $v_{k}(\alpha) \geqslant 3$ and since moreover

$$
v_{2}(\alpha)=2 \frac{n-1}{n-2} \frac{3-\alpha}{2-\alpha} \leqslant 4 \frac{3-\alpha}{2-\alpha}, \quad \text { for } \quad n \geqslant 3
$$

we have

$$
n_{0} \leqslant u_{n}^{*}(\alpha) \leqslant 4 \frac{3-\alpha}{2-\alpha}
$$

and we can take more explicitly in (7)

$$
x_{0}=n n_{0} \frac{\sigma\left(S^{d-1}\right) R^{1-\alpha}}{2-\alpha}
$$

with $n_{0}=1,2,3$, for respectively $n \geqslant 5,13,18$.

5. When $n$ is fixed and $\alpha \nearrow 2$, we have for two constants $K_{1}(n)$ and $K_{2}(n)$

$$
\frac{K_{1}(n)}{(2-\alpha)^{2 /(n+1)}}<\min _{1<k<(n-1+\alpha) / 2} v_{k}(\alpha) \leqslant \frac{K_{2}(n)}{2-\alpha},
$$

so that, from $(12), u_{n}^{*}(\alpha)=u_{n}(\alpha)$ is at most of order $\ln ((n-1) /(2-\alpha))$.

2.1. Lower range for the stable concentration. An estimate on the concentration inequality (1) for small values of $x$ is given by the following theorem.

Theorem 1. Let $X$ be a stable random vector in $\mathbf{R}^{d}$ of index $\alpha \in(1,2)$, and let $f: \mathbf{R}^{d} \rightarrow \mathbf{R}$ be a 1-Lipschitz function. Then for all $n$ large enough and for all

$$
\begin{gathered}
\lambda \in\left(\frac{\sigma\left(S^{d-1}\right)}{\alpha-1}\left(1+\sqrt{\frac{2(\alpha-1)^{2} n \sigma\left(S^{d-1}\right)^{(2-\alpha) /(\alpha-1)}}{\alpha(2-\alpha)}}\right),\right. \\
\left.\frac{\sigma\left(S^{d-1}\right)}{\alpha-1}\left(1+\frac{\alpha-1}{2-\alpha} n u_{n}^{*}(\alpha)\right)\right)
\end{gathered}
$$

there exists $x_{1}(n, \alpha, \lambda)>0$ such that for all $0 \leqslant x \leqslant x_{1}(n, \alpha, \lambda)$,

$$
\begin{aligned}
& \mathbf{P}\{f(X)-\mathbf{E}[f(X)] \geqslant x\} \\
& \leqslant \exp \left(-\frac{2-\alpha}{2 n \sigma\left(S^{d-1}\right)^{1 /(\alpha-1)}}\left(\lambda-\frac{\sigma\left(S^{d-1}\right)}{\alpha-1}\right)^{2}\left(\frac{x}{\lambda}\right)^{\alpha /(\alpha-1)}\right) \\
& \quad+\frac{\sigma\left(S^{d-1}\right)}{\alpha}\left(\frac{x}{\lambda}\right)^{\alpha /(\alpha-1)} \leqslant 1 .
\end{aligned}
$$

In (14), our purpose is to investigate, for $x$ small, the order of concentration from the mean of a Lipschitz function of a stable random vector. The order obtained is essentially $\exp \left(-c x^{\alpha /(\alpha-1)}\right)$ for some explicit constant $c$. Note that the exponent $\alpha /(\alpha-1)$ of $x$ in this bound goes to 2 when $\alpha$ goes 
to 2, this is reminiscent of the Gaussian case. A more precise statement is given in Remark 4 in connection with the Gaussian concentration inequality.

$\mathrm{P} \mathrm{r}$ o o f. In order to investigate concentration for small values of $x$, in this section the level of truncation is chosen by setting $x=\lambda / R^{\alpha-1}$, where $\lambda>0$ is as in (13).

Set also $u(R)=\left(\lambda-\sigma\left(S^{d-1}\right) /(\alpha-1)\right) / R^{\alpha-1}$. Note that $Z_{R}$ has a compound Poisson structure and is the same in law as $Z_{R}=\sum_{k=0}^{N} Z_{k}$, where $Z_{0}=0$, the $Z_{k}, k \geqslant 1$, are i.i.d. random vectors with law $\nu_{Z_{R}} / \nu\{\|u\|>R\}$ and $N$ is an independent Poisson random variable with intensity $\nu\{\|u\|>R\}$. Hence, for any $B \in \mathscr{B}\left(\mathbf{R}^{d}\right)$,

$$
\mathbf{P}\left\{Z_{R} \in B\right\}=\sum_{n=0}^{+\infty} \frac{e^{-\nu\{\|u\|>R\}}}{n !}(\nu\{\|u\|>R\})^{n} \mathbf{P}\left\{\sum_{k=0}^{n} Z_{k} \in B\right\} .
$$

Thus,

$$
\begin{aligned}
\mathbf{E}\left[\left\|Z_{R}\right\|\right] & \leqslant \sum_{n=0}^{+\infty} \frac{e^{-\nu\{\|u\|>R\}}}{n !}(\nu\{\|u\|>R\})^{n} \mathbf{E}\left[\sum_{k=0}^{n}\left\|Z_{k}\right\|\right] \\
& =\mathbf{E}\left[\left\|Z_{1}\right\|\right] e^{-\nu\{\|u\|>R\}} \sum_{n=1}^{+\infty} \frac{\nu\{\|u\|>R\}^{n}}{(n-1) !} \\
& =\mathbf{E}\left[\left\|Z_{1}\right\|\right] \nu\{\|u\|>R\}=\int_{\|u\|>R}\|u\| \nu(d u)=\frac{\sigma\left(S^{d-1}\right)}{\alpha-1} R^{1-\alpha} .
\end{aligned}
$$

Moreover, we also have a lower bound as follows:

$$
\begin{aligned}
\mathbf{E}\left[\left\|Z_{R}\right\|\right] & =\int_{0}^{+\infty} \mathbf{P}\left\{\left\|Z_{R}\right\|>x\right\} d x \\
& =\sum_{n=0}^{+\infty} \frac{e^{-\nu\{\|u\|>R\}}}{n !}(\nu\{\|u\|>R\})^{n} \int_{0}^{+\infty} \mathbf{P}\left\{\left\|\sum_{k=0}^{n} Z_{k}\right\|>x\right\} d x \\
& \geqslant e^{-\nu\{\|u\|>R\}} \nu\{\|u\|>R\} \int_{0}^{+\infty} \mathbf{P}\left\{\left\|Z_{1}\right\|>x\right\} d x \\
& =e^{-\nu\{\|u\|>R\}} \nu\{\|u\|>R\} \mathbf{E}\left[\left\|Z_{1}\right\|\right] \\
& =\frac{\sigma\left(S^{d-1}\right)}{(\alpha-1) R^{\alpha-1}} e^{-\sigma\left(S^{d-1}\right) /\left(\alpha R^{\alpha}\right)} .
\end{aligned}
$$

From (16) and (17), we get

$$
\frac{\sigma\left(S^{d-1}\right)}{(\alpha-1) R^{\alpha-1}} e^{-\sigma\left(S^{d-1}\right) /\left(\alpha R^{\alpha}\right)} \leqslant \mathbf{E}\left[\left\|Z_{R}\right\|\right] \leqslant \frac{\sigma\left(S^{d-1}\right)}{(\alpha-1) R^{\alpha-1}} .
$$

Next, we go back to the study of

$$
\begin{aligned}
\mathbf{P}\{f(X)-\mathbf{E}[f(X)] \geqslant x\} \leqslant & \mathbf{P}\left\{f\left(Y_{R}\right)-\mathbf{E}\left[f\left(Y_{R}\right)\right] \geqslant u(R)\right\} \\
& +\mathbf{P}\left\{Z_{R} \neq 0\right\} .
\end{aligned}
$$


The second summand is estimated as in (5). For the first summand, applying Lemma 1 , we have

$$
\mathbf{P}\left\{f\left(Y_{R}\right)-\mathbf{E}\left[f\left(Y_{R}\right)\right] \geqslant u(R)\right\} \leqslant \exp \left(-\frac{2-\alpha}{2 n \sigma\left(S^{d-1}\right)} \frac{u(R)^{2}}{R^{2-\alpha}}\right),
$$

as long as $0<u(R)<n\left(\sigma\left(S^{d-1}\right) R^{1-\alpha} /(2-\alpha)\right) u_{n}^{*}(\alpha)$, that is, as long as

$$
\left(\lambda-\frac{\sigma\left(S^{d-1}\right)}{\alpha-1}\right) \frac{1}{R^{\alpha-1}}<\frac{n \sigma\left(S^{d-1}\right) u_{n}^{*}(\alpha)}{2-\alpha} R^{1-\alpha},
$$

i.e., for

$$
\lambda<\frac{\sigma\left(S^{d-1}\right)}{\alpha-1}\left(1+\frac{\alpha-1}{2-\alpha} n u_{n}^{*}(\alpha)\right) .
$$

From (19) and (20), we have

$$
\begin{aligned}
\mathbf{P} & \{f(X)-\mathbf{E}[f(X)] \geqslant x\} \\
\leqslant & \exp \left(-\frac{2-\alpha}{2 n \sigma\left(S^{d-1}\right)^{1 /(\alpha-1)}}\left(\lambda-\frac{\sigma\left(S^{d-1}\right)}{\alpha-1}\right)^{2}\left(\frac{x}{\lambda}\right)^{\alpha /(\alpha-1)}\right) \\
& +\frac{\sigma\left(S^{d-1}\right)}{\alpha}\left(\frac{x}{\lambda}\right)^{\alpha /(\alpha-1)} .
\end{aligned}
$$

The bound (22) makes sense if the right-hand side is smaller than 1, this is true at $0^{+}$if

$$
\lambda>\frac{\sigma\left(S^{d-1}\right)}{\alpha-1}+\sqrt{\frac{2 n \sigma\left(S^{d-1}\right)^{\alpha /(\alpha-1)}}{\alpha(2-\alpha)}},
$$

and (21), (23) are compatible if

$$
\frac{2(2-\alpha) \sigma\left(S^{d-1}\right)^{(2-\alpha) /(\alpha-1)}}{\alpha n}<u_{n}^{*}(\alpha)^{2}
$$

which is true if $n$ is large enough or if $\alpha$ is close enough to 2 . Theorem 1 is proved.

$\mathrm{R} \mathrm{e} \mathrm{m} \mathrm{a} \mathrm{r} \mathrm{k} 3$ (comments on the bound and the range of Theorem 1).

1. In fact, the bound (14) holds for all $x>0$ but makes sense only for $x<x_{1}(n, \alpha, \lambda)$.

2. The value $x_{1}(n, \alpha, \lambda)$ in the bound of the domain of Theorem 1 can be made more precise: $x_{1}(n, \alpha, \lambda)=\lambda u_{0}(n, \alpha, \lambda)^{(\alpha-1) / \alpha}$, where $u_{0}(n, \alpha, \lambda)$ is a unique positive solution of

$$
\exp \left(-\frac{2-\alpha}{2 n \sigma\left(S^{d-1}\right)^{1 /(\alpha-1)}}\left(\lambda-\frac{\sigma\left(S^{d-1}\right)}{\alpha-1}\right)^{2} u\right)+\frac{\sigma\left(S^{d-1}\right)}{\alpha} u=1 .
$$


In particular, we have

$$
\begin{aligned}
u_{0}(n, \alpha, \lambda) \geqslant & \frac{2 n \sigma\left(S^{d-1}\right)^{1 /(\alpha-1)}}{(2-\alpha)\left(\lambda-\sigma\left(S^{d-1}\right) /(\alpha-1)\right)^{2}} \\
& \times \ln \left(\frac{\alpha(2-\alpha)}{2 n \sigma\left(S^{d-1}\right)^{\alpha /(\alpha-1)}}\left(\lambda-\frac{\sigma\left(S^{d-1}\right)}{\alpha-1}\right)^{2}\right),
\end{aligned}
$$

so that we can take

$$
\begin{aligned}
x_{1}(n, \alpha, \lambda)=\lambda & \sigma\left(S^{d-1}\right)^{1 / \alpha} \\
\times & \left(\frac{2 n}{(2-\alpha)\left(\lambda-\sigma\left(S^{d-1}\right) /(\alpha-1)\right)^{2}}\right. \\
& \left.\times \ln \left(\frac{\alpha(2-\alpha)}{2 n \sigma\left(S^{d-1}\right)^{\alpha /(\alpha-1)}}\left(\lambda-\frac{\sigma\left(S^{d-1}\right)}{\alpha-1}\right)^{2}\right)\right)^{(\alpha-1) / \alpha} .
\end{aligned}
$$

3. In order to optimize the bound (14) with respect to $\lambda$, observe that the first summand (which gives the right order) gives the best bound for

$$
\lambda=\lambda_{0}(\alpha):=\frac{\alpha \sigma\left(S^{d-1}\right)}{(2-\alpha)(\alpha-1)} .
$$

Denoting by $\left(\lambda_{1}(n, \alpha), \lambda_{2}(n, \alpha)\right)$ the interval in (13), we have $\lambda_{0}(\alpha) \geqslant$ $\lambda_{1}(n, \alpha)$ if $n \sigma\left(S^{d-1}\right)^{(2-\alpha) /(\alpha-1)} \leqslant 2 \alpha /(\alpha-1)$ and $\lambda_{0}(\alpha) \leqslant \lambda_{2}(n, \alpha)$ if $n u_{n}^{*}(\alpha) \geqslant 2$, so that in general $\lambda_{0}(\alpha) \leqslant \lambda_{1}(n, \alpha)$, and the best order should thus occurs for $\lambda=\lambda_{1}(n, \alpha)$. But for this choice $x_{1}(n, \alpha, \lambda)=0$ and the domain of validity of the concentration inequality is empty. Thus, there is no optimal choice for $\lambda \in\left(\lambda_{1}(n, \alpha), \lambda_{2}(n, \alpha)\right)$ : on the one hand, $\lambda=\lambda_{1}(n, \alpha)$ achieves the best concentration rate but in this case the domain is empty; on the other hand, $\lambda=\lambda_{2}(n, \alpha)$ realizes the largest domain but in this case the concentration inequality is the worst. See Remark 5 below for a result without extra parameter.

4. In (14), the exponential is the main term of the bound. For any $\varepsilon>0$, we thus can rewrite (14): for $x$ small enough

$$
\begin{aligned}
& \mathbf{P}\{f(X)-\mathbf{E}[f(X)] \geqslant x\} \\
& \quad \leqslant(1+\varepsilon) \exp \left(-\frac{2-\alpha}{2 n \sigma\left(S^{d-1}\right)^{1 /(\alpha-1)}}\left(\lambda-\frac{\sigma\left(S^{d-1}\right)}{\alpha-1}\right)^{2}\left(\frac{x}{\lambda}\right)^{\alpha /(\alpha-1)}\right) .
\end{aligned}
$$

Thus, it gives the order of stable concentration for small values of $x$ : roughly speaking, it is in $\exp \left(-c x^{\alpha /(\alpha-1)}\right)$.

$\mathrm{R}$ e $\mathrm{m}$ a r k 4 (Gaussian result). We can recover a suboptimal Gaussian result from the bound (14) by letting $\alpha$ go to 2 in a proper manner. To do so, and following [4], consider a stable random vector $X^{(\alpha)}$ whose Lévy measure has for spherical component $\sigma$, the uniform measure with total mass $\sigma\left(S^{d-1}\right)=d(2-\alpha)$. Now, as $\alpha$ goes to 2 , the vector $X^{(\alpha)}$ converges 
in distribution to a standard normal random vector $W$. Moreover, note that when letting $\alpha \rightarrow 2$ with $\sigma\left(S^{d-1}\right)=d(2-\alpha)$ in (21) and (25), the ranges for $\lambda$ and for $x$ in Theorem 1 become $\lambda \in(0, \infty)$ and $x \in(0, \infty)$ while the bound (14) becomes $\exp \left(-x^{2} /(2 d n)\right)$. This is not the classical bound for Gaussian concentration, since it depends on $d$ and $n$. But, first note that we could replace $n$ large in Theorem 1 by $\alpha$ close enough to 2 (see inequality (24) in the proof). Then applying Lemma 1 with arbitrary $\delta>0$ and taking the limit $\alpha \rightarrow 2$ in (14), we obtain similarly

$$
\mathbf{P}\{f(W)-\mathbf{E}[f(W)] \geqslant x\} \leqslant \exp \left(-\frac{x^{2}}{2 d n_{\delta}}\right),
$$

for any $x>0$. Finally, letting $\delta \rightarrow 0$ yields for all $x>0$ :

$$
\mathbf{P}\{f(W)-\mathbf{E}[f(W)] \geqslant x\} \leqslant \exp \left(-\frac{x^{2}}{2 d}\right) .
$$

This last result is indeed suboptimal, since it depends on the dimension $d$. Another approximation procedure would be to take for spherical part, the weighted sum of the Dirac measures at the points $(0, \ldots, 0, \pm 1,0, \ldots, 0)$, with weight all equal to $(2-\alpha) / 2$. The components of $X^{(\alpha)}$ are thus independent and when $\alpha$ goes to 2 , the vector $X^{(\alpha)}$ converges in distribution to a standard normal random vector $W$.

The same type of argument can be applied to the intermediate regime of concentration studied in Section 2.2, see Remark 8 .

$\mathrm{R}$ e $\mathrm{mark} 5$. We can give a bound for small values of $x$ without introducing an extra parameter $\lambda$ as in Theorem 1. To do so, let $\varepsilon>0$. For all $n \geqslant 5$ (or for $\alpha$ close enough to 2), there exists $x_{0}(n, \varepsilon)>0$ such that for all $0 \leqslant x<x_{0}(n, \varepsilon)$

$$
\begin{aligned}
& \mathbf{P}\{f(X)-\mathbf{E}[f(X)] \geqslant x\} \\
& \quad \leqslant(1+\varepsilon) \exp \left(-\frac{2-\alpha}{2 n \sigma\left(S^{d-1}\right)^{1 /(\alpha-1)}}\left(\frac{\alpha-1}{\alpha}\right)^{\alpha /(\alpha-1)} x^{\alpha /(\alpha-1)}\right) .
\end{aligned}
$$

The drawback of this bound is in its range, since we do not know explicitly $x_{0}(n, \varepsilon)$.

The proof of (26) follows the same lines of reasoning as that of Theorem 1 but with the level of truncation chosen by setting $x=\alpha \mathbf{E}\left[\left\|Z_{R}\right\|\right]$. Set also

$$
u(R)=(\alpha-1) \mathbf{E}\left[\left\|Z_{R}\right\|\right]
$$

and study the summands of (19). Applying Lemma 1 and using

$$
\frac{u(R)^{2}}{R^{2-\alpha}}=\left(\frac{\alpha-1}{\alpha}\right)^{2} \frac{x^{2}}{R^{2-\alpha}} \geqslant\left(\frac{1}{\sigma\left(S^{d-1}\right)}\right)^{(2-\alpha) /(\alpha-1)}\left(\frac{\alpha-1}{\alpha}\right)^{\alpha /(\alpha-1)} x^{\alpha /(\alpha-1)}
$$


the first summand in the right-hand side of (19) is bounded as follows:

$$
\begin{aligned}
& \mathbf{P}\left\{f\left(Y_{R}\right)-\mathbf{E}\left[f\left(Y_{R}\right)\right] \geqslant u(R)\right\} \\
& \quad \leqslant \exp \left(-\frac{2-\alpha}{2 n \sigma\left(S^{d-1}\right)^{1 /(\alpha-1)}}\left(\frac{\alpha-1}{\alpha}\right)^{\alpha /(\alpha-1)} x^{\alpha /(\alpha-1)}\right),
\end{aligned}
$$

as long as $0<\left((\alpha-1) \alpha^{-1}\right) x=u(R)<n \sigma\left(S^{d-1}\right) R^{1-\alpha}(2-\alpha)^{-1} u_{n}^{*}(\alpha)$, i.e., as long as

$$
\mathbf{E}\left[\left\|Z_{R}\right\|\right]<\frac{n \sigma\left(S^{d-1}\right) u_{n}^{*}(\alpha)}{(2-\alpha)(\alpha-1)} R^{1-\alpha} .
$$

It follows from the right-hand side of (18) that (27) is true if $\sigma\left(S^{d-1}\right) /$ $(\alpha-1)<n \sigma\left(S^{d-1}\right) u_{n}^{*}(\alpha) /((2-\alpha)(\alpha-1))$, i.e., if $n u_{n}^{*}(\alpha)>2-\alpha$, which is true at least for $\alpha$ close enough to 2 or for $n \geqslant 5$, since then $u_{n}^{*}(\alpha) \geqslant 1$. Using (5) for the second summand on the right-hand side of (19), we derive

$$
\begin{aligned}
& \mathbf{P}\{f(X)-\mathbf{E}[f(X)] \geqslant x\} \\
& \quad \leqslant \exp \left(-\frac{2-\alpha}{2 n \sigma\left(S^{d-1}\right)^{1 /(\alpha-1)}}\left(\frac{\alpha-1}{\alpha}\right)^{\alpha /(\alpha-1)} x^{\alpha /(\alpha-1)}\right)+\frac{\sigma\left(S^{d-1}\right)}{\alpha R^{\alpha}} .
\end{aligned}
$$

But from (18), when $x$ goes to 0 , we have $R \rightarrow+\infty$ or $R \rightarrow 0$. Moreover, when $x \rightarrow 0, \mathbf{E}\left[\left\|Z_{R}\right\|\right] \rightarrow 0$, so that $Z_{R} \rightarrow 0$ in $L^{1}\left(\mathbf{R}^{d}\right)$ and $Z_{R} \Rightarrow \delta_{0}$. This implies the convergence of $\nu_{Z_{R}}=\nu_{B(0, R)^{c}}$ to 0 , from which we get $R \rightarrow+\infty$. Thus when $x$ is small, the main term in the bound (28) is given by the exponential. Since the second term goes to 0 , for any $\varepsilon>0$, there is some $x_{0}(n, \varepsilon)$ such that for $x \leqslant x_{0}(n, \varepsilon)$ the bound (26) holds true.

2.2. Intermediate range for the stable concentration. In this section, we study (1) for intermediate values of $x$. To do so, the level of truncation is changed in (3). Moreover, in order to derive a Gaussian inequality as a limiting stable case, we shall use Lemma 1 with the parameter $\delta>0$. In the limiting case, $\delta$ will go to 0 . First, we state the following theorem.

Theorem 2. Let $X$ be a stable random vector in $\mathbf{R}^{d}$ of index $\alpha \in(1,2)$, and let $f: \mathbf{R}^{d} \rightarrow \mathbf{R}$ be a 1-Lipschitz function. Let $\delta>0$ and $n \in \mathbb{N}, n \geqslant 2$. Set $n_{\delta}=1+\delta(n-1)$. Then for any $\varepsilon$ satisfying

$$
\varepsilon>\frac{(2-\alpha) e}{2 \alpha n_{\delta}},
$$

we have

$$
\begin{aligned}
& \mathbf{P}\{f(X)-\mathbf{E}[f(X)] \geqslant x\} \leqslant(1+\varepsilon) \\
& \quad \times \exp \left(-\frac{2-\alpha}{2 n_{\delta} \sigma\left(S^{d-1}\right)}\left(x\left(1+\frac{2-\alpha}{2 n_{\delta}(\alpha-1) u_{1}\left(n_{\delta}, \alpha\right)}\right)^{-1}\right)^{\alpha}\right),
\end{aligned}
$$


for any $x$ such that

$$
\begin{aligned}
& \frac{2 n_{\delta} \sigma\left(S^{d-1}\right)}{2-\alpha} u_{1}\left(n_{\delta}, \alpha\right)\left(1+\frac{2-\alpha}{2 n_{\delta}(\alpha-1) u_{1}\left(n_{\delta}, \alpha\right)}\right)^{\alpha}<x^{\alpha} \\
& <\frac{2 n_{\delta} \sigma\left(S^{d-1}\right)}{2-\alpha}\left(u_{2}\left(n_{\delta}, \alpha\right) \wedge \frac{u_{n}^{*}(\alpha, \delta)}{2}\right) \\
& \quad \times\left(1+\frac{2-\alpha}{2 n(\alpha-1)\left(u_{2}\left(n_{\delta}, \alpha\right) \wedge u_{n}^{*}(\alpha, \delta) / 2\right)}\right)^{\alpha},
\end{aligned}
$$

where $u_{1}\left(n_{\delta}, \alpha\right)$ and $u_{2}\left(n_{\delta}, \alpha\right)$ are the solutions of

$$
e^{u}-\frac{2 n_{\delta} \alpha \varepsilon}{2-\alpha} u=0
$$

and $u_{n}^{*}(\alpha, \delta)$ is given by $(6)$.

$\mathrm{P} \mathrm{r}$ o o f. First, using the right-hand side of (18), the bound (3) becomes

$$
\begin{aligned}
& \mathbf{P}\{f(X)-\mathbf{E}[f(X)] \geqslant x\} \\
& \quad \leqslant \mathbf{P}\left\{f\left(Y_{R}\right)-\mathbf{E}\left[f\left(Y_{R}\right)\right] \geqslant x-\frac{\sigma\left(S^{d-1}\right)}{\alpha-1} R^{1-\alpha}\right\}+\mathbf{P}\left\{Z_{R} \neq 0\right\} .
\end{aligned}
$$

Next choose the level of truncation by setting

$$
R\left(1+\frac{\sigma\left(S^{d-1}\right)}{(\alpha-1) R^{\alpha}}\right)=x
$$

We thus have

$$
\mathbf{P}\{f(X)-\mathbf{E}[f(X)] \geqslant x\} \leqslant \mathbf{P}\left\{f\left(Y_{R}\right)-\mathbf{E}\left[f\left(Y_{R}\right)\right] \geqslant R\right\}+\mathbf{P}\left\{Z_{R} \neq 0\right\} .
$$

Applying Lemma 1 with $x=R$ in (8) to estimate the first summand on the right-hand side of (33) and using (5) for the second one, the bound (33) then becomes

$$
\mathbf{P}\{f(X)-\mathbf{E}[f(X)] \geqslant x\} \leqslant \exp \left(-\frac{(2-\alpha) R^{\alpha}}{2 n_{\delta} \sigma\left(S^{d-1}\right)}\right)+\frac{\sigma\left(S^{d-1}\right)}{\alpha R^{\alpha}}
$$

as long as, using (7) with $x=R$,

$$
R^{\alpha} \leqslant n_{\delta} \frac{\sigma\left(S^{d-1}\right)}{2-\alpha} u_{n}^{*}(\alpha, \delta) .
$$

Next, we compare the two summands on the right-hand side of (34). To do so, set $u=(2-\alpha) R^{\alpha} /\left(2 n_{\delta} \sigma\left(S^{d-1}\right)\right)$ and let us compare $(2-\alpha) /(2 n \alpha u)$ to $e^{-u}$ by studying the function

$$
h_{n, \alpha}(u)=e^{u}-\frac{2 n_{\delta} \alpha \varepsilon}{2-\alpha} u
$$


Note that $h_{n, \alpha}$ has a unique minimum at $u_{0}=\ln \left(2 n_{\delta} \alpha \varepsilon /(2-\alpha)\right)$, which is negative because of (29). We thus have

$$
\mathbf{P}\{f(X)-\mathbf{E}[f(X)] \geqslant x\} \leqslant(1+\varepsilon) \exp \left(-\frac{2-\alpha}{2 n_{\delta} \sigma\left(S^{d-1}\right)} R^{\alpha}\right),
$$

for

$$
\frac{2 n_{\delta} \sigma\left(S^{d-1}\right)}{2-\alpha} u_{1}\left(n_{\delta}, \alpha\right)<R^{\alpha}<\frac{2 n_{\delta} \sigma\left(S^{d-1}\right)}{2-\alpha} u_{2}\left(n_{\delta}, \alpha\right),
$$

and still under the condition (35), with moreover $R$ given by the relation (32).

We now express (37) and the associated conditions (35) and (38) in terms of $x$. Since from (32)

$$
R \leqslant x \leqslant R\left(1+\frac{2-\alpha}{2 n_{\delta}(\alpha-1) u_{1}\left(n_{\delta}, \alpha\right)}\right),
$$

the bound (37) becomes (30).

To express the range in terms of $x$, note that we have $x=\theta\left(R^{\alpha}\right)$ with

$$
\theta(u)=u^{1 / \alpha}\left(1+\frac{\sigma\left(S^{d-1}\right)}{(\alpha-1) u}\right)
$$

The function $\theta$ is an increasing bijection from $\left[\sigma\left(S^{d-1}\right),+\infty\right)$ to $[(\alpha /(\alpha-$ 1)) $\left.\sigma\left(S^{d-1}\right)^{1 / \alpha},+\infty\right)$

Then, note that $\sigma\left(S^{d-1}\right) \leqslant\left(2 n_{\delta} \sigma\left(S^{d-1}\right) /(2-\alpha)\right) u_{1}\left(n_{\delta}, \alpha\right)$, i.e., $(2-$ $\alpha) /\left(2 n_{\delta}\right) \leqslant u_{1}\left(n_{\delta}, \alpha\right)$, which is equivalent to have $h_{n, \alpha}\left((2-\alpha) /\left(2 n_{\delta}\right)\right) \geqslant 0$ and $h_{n, \alpha}^{\prime}\left((2-\alpha) /\left(2 n_{\delta}\right)\right) \leqslant 0$. Indeed, writing $\varepsilon=(2-\alpha) \widetilde{e} /\left(2 n_{\delta} \alpha\right)$ in $(29)$, with some $\tilde{e}>e$, we have

$$
h_{n, \alpha}\left(\frac{2-\alpha}{2 n_{\delta}}\right)=\exp \left(\frac{2-\alpha}{2 n_{\delta}}\right)-\alpha \varepsilon=\exp \left(\frac{2-\alpha}{2 n_{\delta}}\right)-\frac{2-\alpha}{2 n_{\delta}} \widetilde{e} .
$$

Let $\eta(u)=e^{u}-\tilde{e} u$. Since $(2-\alpha) /\left(2 n_{\delta}\right) \leqslant \frac{1}{2}<1$, we have $\eta((2-$ $\left.\alpha) /\left(2 n_{\delta}\right)\right)>0$ as long as $\tilde{e}$ is such that $\tilde{e} \leqslant 2 \sqrt{e}$. We thus deduce that $h_{n, \alpha}\left((2-\alpha) /\left(2 n_{\delta}\right)\right) \geqslant 0$.

Next,

$$
h_{n, \alpha}^{\prime}\left(\frac{2-\alpha}{2 n_{\delta}}\right)=\exp \left(\frac{2-\alpha}{2 n_{\delta}}\right)-\frac{2 n_{\delta} \alpha}{2-\alpha} \varepsilon \leqslant \exp \left(\frac{2-\alpha}{2 n_{\delta}}\right)-e,
$$

which is nonpositive, since $2-\alpha \leqslant 2 n_{\delta}$. Finally,

$$
\sigma\left(S^{d-1}\right) \leqslant \frac{2 n_{\delta} \sigma\left(S^{d-1}\right)}{2-\alpha} u_{1}\left(n_{\delta}, \alpha\right)
$$

Using $\theta$ in the domain, where it defines an increasing bijection, the conditions (35) and (38) can be rewritten as:

$$
\theta\left(\frac{2 n_{\delta} \sigma\left(S^{d-1}\right)}{2-\alpha} u_{1}\left(n_{\delta}, \alpha\right)\right)<x<\theta\left(\frac{2 n_{\delta} \sigma\left(S^{d-1}\right)}{2-\alpha}\left(u_{2}\left(n_{\delta}, \alpha\right) \wedge \frac{u_{n}^{*}(\alpha, \delta)}{2}\right)\right) .
$$


The range of (30) is thus (31) when $\varepsilon$ is such that

$$
\frac{(2-\alpha) e}{2 n_{\delta} \alpha}<\varepsilon<\frac{(2-\alpha) \sqrt{e}}{n_{\delta} \alpha} \text {. }
$$

Finally, it is clear that if (30) holds for some $\varepsilon>0$, it also holds for any larger $\varepsilon$. The condition on $\varepsilon$ can thus be reduced to (29). Theorem 2 is proved.

$\mathrm{R}$ e $\mathrm{m}$ a $\mathrm{rk}$ 6. 1. From (29), note that

$$
h_{n, \alpha}\left(\ln \left(\frac{2 n_{\delta} \alpha \varepsilon}{2-\alpha}\right)\right)=\frac{2 n_{\delta} \alpha \varepsilon}{2-\alpha}\left(1-\ln \left(\frac{2 n_{\delta} \alpha \varepsilon}{2-\alpha}\right)\right)<0,
$$

so that $u_{2}\left(n_{\delta}, \alpha\right)>\ln \left(2 n_{\delta} \alpha \varepsilon /(2-\alpha)\right)>1$.

2. The parameter $n \geqslant 2$ is free to vary in Theorem 2. Thus changing $n$ allows us to shift the range (31) so that we can derive a concentration bound of the type $e^{-c x^{\alpha}}$ on a larger domain, by eventually changing $n$ and the generic constant $c$.

$\mathrm{R}$ e $\mathrm{m}$ a r k 7 (comments on the bounds in the domain (31)). It is interesting to investigate the behavior of $\left(2 n_{\delta} \alpha /(2-\alpha)\right) u_{i}\left(n_{\delta}, \alpha\right), i=1,2$, when $\alpha$ goes to 2 or when $n$ goes to $+\infty$ in the domain given by (31).

To simplify notation, (36) is written as $h_{a}(u)=e^{u}-(\varepsilon / a) u$ with $\varepsilon>a e$, and without loss of generality, we study the behavior of the ze$\operatorname{ros} u_{1}(a)<u_{2}(a)$ of $h_{a}(u)$ as $a \rightarrow 0$.

Since $h_{a}(\ln (\varepsilon / a))<0$ and $\lim _{a \rightarrow 0} h_{a}\left(a^{\lambda}\right)=1-\lambda \varepsilon$, for $\lambda<\varepsilon<\tilde{\lambda}$, we have $\lambda a \leqslant u_{1}(a) \leqslant \tilde{\lambda} a \leqslant \ln (\varepsilon / a) \leqslant u_{2}(a)$. Thus,

$$
\lim _{a \rightarrow 0} \frac{u_{1}(a)}{a}=\varepsilon, \quad \lim _{a \rightarrow 0} \frac{u_{2}(a)}{a}=+\infty,
$$

i.e., in our setting

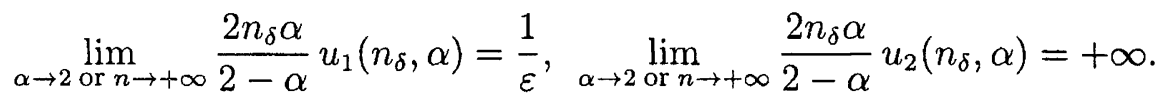

For $\alpha$ close to 2 or for $n$ large enough, $1+(2-\alpha) /\left(2 n_{\delta}(\alpha-1) u_{1}\left(n_{\delta}, \alpha\right)\right) \rightarrow$ $1+\alpha \varepsilon /(\alpha-1)$, so that for $\varepsilon<1$ and for $\alpha$ close to 2 or for $n$ large enough, $1+(2-\alpha) /\left(2 n_{\delta}(\alpha-1) u_{1}\left(n_{\delta}, \alpha\right)\right) \leqslant 1+\alpha /(\alpha-1)$. In this case, $(30)$ becomes $\mathbf{P}\{f(X)-\mathbf{E}[f(X)] \geqslant x\} \leqslant(1+\varepsilon) \exp \left(-\frac{2-\alpha}{2 n_{\delta} \sigma\left(S^{d-1}\right)}\left(\frac{x}{1+\alpha /(\alpha-1)}\right)^{\alpha}\right)$, and the range (31) reduces to

$$
\begin{aligned}
& \left(1+\frac{\alpha \varepsilon}{\alpha-1}\right)^{\alpha} \frac{\sigma\left(S^{d-1}\right)}{\alpha \varepsilon}<x^{\alpha} \\
& \quad< \begin{cases}\frac{2 n_{\delta} \sigma\left(S^{d-1}\right)}{2-\alpha} \ln \left(\sqrt{\frac{n_{\delta}-1}{2-\alpha}}\right) & \text { if } \alpha \rightarrow 2, \\
\frac{3 n_{\delta} \sigma\left(S^{d-1}\right)}{2-\alpha} & \text { if } n \rightarrow+\infty .\end{cases}
\end{aligned}
$$


$\mathrm{R}$ e $\mathrm{m}$ a r k 8 (more on the Gaussian results). 1. When $\alpha$ is close to 2, as in Remark 4, we recover (i) of Theorem 2 in [4] and in particular still following [4], we recover a suboptimal Gaussian inequality. Indeed, doing the same when $\alpha \rightarrow 2$, we obtain

$$
\mathbf{P}\{f(W)-\mathbf{E}[f(W)] \geqslant x\} \leqslant(1+\varepsilon) \exp \left(-\frac{1}{2 d n_{\delta}}\left(\frac{x}{1+\varepsilon}\right)^{2}\right) .
$$

This holds true for any $x>0$, since the left-hand side of (31) goes to 0 when $\alpha$ goes to $2\left(u_{1}\left(n_{\delta}, \alpha\right) \rightarrow 0\right.$ as $\left.\alpha \rightarrow 2\right)$ while its right-hand side goes to $+\infty\left(u_{2}\left(n_{\delta}, \alpha\right) \rightarrow+\infty\right.$ and $u_{n}^{*}(\alpha, \delta) \rightarrow+\infty$ as $\left.\alpha \rightarrow 2\right)$. Finally, letting $\varepsilon \rightarrow 0$ and $\delta \rightarrow 0$ yields for all $x>0$ :

$$
\mathbf{P}\{f(W)-\mathbf{E}[f(W)] \geqslant x\} \leqslant \exp \left(-\frac{x^{2}}{2 d}\right) .
$$

2. Note also that letting $\delta \rightarrow 0$ in the range (31) does not allow one to recover a concentration result for arbitrarily small values of $x$. Theorem 1 cannot thus be derived from Theorem 2 and vice versa.

2.3. Another intermediate range for stable concentration. The concentration and the range obtained in Section 2.2 depend on $u_{1}\left(n_{\delta}, \alpha\right)$ which is not explicitly given. In this section, using directly (4) rather than the bound (5), we obtain the same type of result, probably less sharp, but with more explicit bounds.

Theorem 3. Let $X$ be a stable random vector in $\mathbf{R}^{d}$ of index $\alpha \in(1,2)$, and let $f: \mathbf{R}^{d} \rightarrow \mathbf{R}$ be a 1-Lipschitz function. Then for any $\varepsilon$ satisfying

$$
\varepsilon \geqslant \frac{(2-\alpha) e}{2 n \alpha},
$$

we have

$$
\begin{aligned}
\mathbf{P}\{f(X)-\mathbf{E}[f(X)] \geqslant x\} & \\
\leqslant(1+\varepsilon) \exp ( & -\frac{2-\alpha}{2 n \sigma\left(S^{d-1}\right)} \\
& \left.\times\left(x\left(1+\frac{2-\alpha}{2 n(\alpha-1)\left(1-((2-\alpha) /(2 n \alpha))^{2 / 3}\right)}\right)^{-1}\right)^{\alpha}\right)
\end{aligned}
$$

for all $x$ such that

$$
\begin{aligned}
& \frac{2 n \sigma\left(S^{d-1}\right)}{2-\alpha}\left(1-\left(\frac{2-\alpha}{2 n \alpha}\right)^{2 / 3}\right)\left(1+\frac{2-\alpha}{2 n(\alpha-1)\left(1-((2-\alpha) /(2 n \alpha))^{2 / 3}\right)}\right)^{\alpha} \\
& <x^{\alpha}<\frac{2 n \sigma\left(S^{d-1}\right)}{2-\alpha}\left(\left(1+\left(\left(\frac{2-\alpha}{2 n \alpha}\right)^{2 / 3} \wedge 0.68\right)\right) \wedge \frac{u_{n}^{*}(\alpha)}{2}\right) \\
& \quad \times\left(1+\frac{2-\alpha}{2 n(\alpha-1)\left(\left(1+\left(((2-\alpha) /(2 n \alpha))^{2 / 3} \wedge 0.68\right)\right) \wedge u_{n}^{*}(\alpha) / 2\right)}\right)^{\alpha},
\end{aligned}
$$

where $u_{n}^{*}(\alpha)$ is given by $(6)$ with $\delta=1$. 
P r o o f. With (4), inequality (33) becomes

$$
\begin{aligned}
& \mathbf{P}\{f(X)-\mathbf{E}[f(X)] \geqslant x\} \\
& \quad \leqslant \exp \left(-\frac{(2-\alpha) R^{\alpha}}{2 n \sigma\left(S^{d-1}\right)}\right)+1-\exp \left(-\frac{\sigma\left(S^{d-1}\right)}{\alpha R^{\alpha}}\right),
\end{aligned}
$$

as long as (35) holds (once more using (7) with $y=R$ ).

We next compare the two summands on the right-hand side of (44). To do so, let $u=(2-\alpha) R^{\alpha} /\left(2 n \sigma\left(S^{d-1}\right)\right)$ and let $c=(2-\alpha) /(2 n \alpha)$ and thus we compare $1-e^{-c / u}$ and $e^{-u}$. To this end, let us study for some $\varepsilon>0$ the function

$$
g_{n, \alpha}(u)=\varepsilon e^{-u}+e^{-c / u},
$$

and in particular let us see when it is larger than 1 . We cannot study the function $g_{n, \alpha}$ in (45) as easily as the function $h_{n, \alpha}$ in (36), the argument is thus different from that of Section 2.2. However (41) guarantees that $g_{n, \alpha}$ takes values greater than 1 , since $g_{n, \alpha}(1) \geqslant c+e^{-c} \geqslant 1$, for all $c>0$. We investigate now whether or not, for some interval containing 1 the function $g_{n, \alpha}$ is larger than 1 . Since $\varepsilon \geqslant e c$, we look for $c e^{1-u}+e^{-c / u} \geqslant 1$.

Observe first that for some fixed $u$, the function $c \rightarrow c e^{1-u}+e^{-c / u} \geqslant 1$ is increasing for $c \geqslant c_{0}(u)=u(u-1)-u \ln u>0$.

Elementary computations show that $|u-1|^{3 / 2} \geqslant c_{0}(u)$ for all $u \leqslant u_{1} \simeq 3.2$ and $|u-1|^{3 / 2} e^{1-u}+e^{-|u-1|^{3 / 2} / u} \geqslant 1$ for $0 \leqslant u \leqslant u_{2} \simeq 1.68$. Next, for $\varepsilon \geqslant e c,|u-1|^{3 / 2} \leqslant c$, and $u \leqslant u_{2}$, we have

$$
1 \leqslant|u-1|^{3 / 2} e^{1-u}+e^{-|u-1|^{3 / 2} / u} \leqslant c e^{1-u}+e^{-c / u} \leqslant \varepsilon e^{1-u}+e^{-c / u} .
$$

Thus, we conclude that

$$
1-\exp \left(-\frac{\sigma\left(S^{d-1}\right)}{\alpha R^{\alpha}}\right) \leqslant \varepsilon \exp \left(-\frac{(2-\alpha) R^{\alpha}}{2 n \sigma\left(S^{d-1}\right)}\right)
$$

for $R$ in the range

$$
\begin{aligned}
0 & <\frac{2 n \sigma\left(S^{d-1}\right)}{2-\alpha}\left(1-\left(\frac{2-\alpha}{2 n \alpha}\right)^{2 / 3}\right)<R^{\alpha} \\
& <\frac{2 n \sigma\left(S^{d-1}\right)}{2-\alpha}\left(1+\left(\left(\frac{2-\alpha}{2 n \alpha}\right)^{2 / 3} \wedge 0.68\right)\right) .
\end{aligned}
$$

Hence, we have obtained a mobile range of varying length for which we have

$$
\mathbf{P}\{f(X)-\mathbf{E}[f(X)] \geqslant x\} \leqslant(1+\varepsilon) \exp \left(-\frac{2-\alpha}{2 n \sigma\left(S^{d-1}\right)} R^{\alpha}\right) .
$$

Next, (47) and its conditions (35) and (46) can be expressed in terms of $x$. Since

$$
R \leqslant x \leqslant R\left(1+\frac{2-\alpha}{2 n(\alpha-1)\left(1-((2-\alpha) /(2 n \alpha))^{2 / 3}\right)}\right),
$$

(42) is obtained by using the bound (47). 
To express the range in terms of $x$, argue as in the proof of Theorem 2 using the function $\theta$. Note that

$$
\sigma\left(S^{d-1}\right) \leqslant \frac{2 n \sigma\left(S^{d-1}\right)}{2-\alpha}\left(1-\left(\frac{2-\alpha}{2 n \alpha}\right)^{2 / 3}\right)
$$

indeed this is saying that

$$
\frac{2-\alpha}{2 n} \leqslant 1-\left(\frac{2-\alpha}{2 n \alpha}\right)^{2 / 3}
$$

which is clearly true, since

$$
\frac{2-\alpha}{2 n \alpha} \leqslant \frac{1}{4} \quad \text { and } \quad \alpha u \leqslant 2 u \leqslant 1-u^{2 / 3}
$$

for all $0 \leqslant u \leqslant \frac{1}{4}$.

We can thus use the function $\theta$ in the domain, where it defines an increasing bijection, the conditions (35) and (46) can be rewritten as:

$$
\begin{aligned}
& \theta\left(\frac{2 n \sigma\left(S^{d-1}\right)}{2-\alpha}\left(1-\left(\frac{2-\alpha}{2 n \alpha}\right)^{2 / 3}\right)\right) \\
& \quad<x<\theta\left(\frac{2 n \sigma\left(S^{d-1}\right)}{2-\alpha}\left(\left(1+\left(\left(\frac{2-\alpha}{2 n \alpha}\right)^{2 / 3} \wedge 0.68\right)\right) \wedge \frac{u_{n}^{*}(\alpha)}{2}\right)\right)
\end{aligned}
$$

from which we obtain the range (43). Theorem 3 is proved.

$\mathrm{R}$ e $\mathrm{m}$ a r k 9 (comments on the bounds in the domain (43)). We discuss below the behavior of the domain (43) as $\alpha$ goes to 2 or as $n$ goes to $+\infty$. Let $\eta>0$. Since for $\alpha$ close enough to 2 or $n$ large enough

$$
\left(1+\frac{2-\alpha}{2 n(\alpha-1)\left(1-((2-\alpha) /(2 n \alpha))^{2 / 3}\right)}\right)^{\alpha} \leqslant 1+\eta,
$$

inequality (42) can be rewritten as

$$
\mathbf{P}\{f(X)-\mathbf{E}[f(X)] \geqslant x\} \leqslant(1+\varepsilon) \exp \left(-\frac{2-\alpha}{2(1+\eta) n \sigma\left(S^{d-1}\right)} x^{\alpha}\right) .
$$

Moreover, since

$$
\left(1+\frac{2-\alpha}{2 n(\alpha-1)\left(\ln (2 n /(2-\alpha)) \wedge u_{n}^{*}(\alpha) / 2\right)}\right)^{\alpha} \geqslant 1,
$$

the domain in (43) can be replaced, for $\alpha$ close enough to 2 or for $n$ large enough, by

$$
\begin{aligned}
& \frac{2(1+\eta) n \sigma\left(S^{d-1}\right)}{2-\alpha}\left(1-\left(\frac{2-\alpha}{2 n \alpha}\right)^{2 / 3}\right)<x^{\alpha} \\
& <\frac{2 n \sigma\left(S^{d-1}\right)}{2-\alpha}\left(\left(1+\left(\frac{2-\alpha}{2 n \alpha}\right)^{2 / 3}\right) \wedge \frac{u_{n}^{*}(\alpha)}{2}\right) .
\end{aligned}
$$


However, we have seen that for $\alpha$ close enough to $2, u_{n}^{*}(\alpha)=u_{n}(\alpha)$ satisfies (12), so that we obtain the following domain with different orders in the lower and upper bounds in $n$ or $\alpha$ :

$$
\frac{2(1+\eta) n \sigma\left(S^{d-1}\right)}{2-\alpha}\left(1-\left(\frac{2-\alpha}{2 n \alpha}\right)^{2 / 3}\right)<x^{\alpha}<\frac{2 n \sigma\left(S^{d-1}\right)}{2-\alpha} \ln \left(\sqrt{\frac{n-1}{2-\alpha}}\right) .
$$

For $n$ large enough, $u_{n}^{*}(\alpha) \in[3,4(3-\alpha) /(2-\alpha)]$ is bounded so that the minimum in (48) is $u_{n}^{*}(\alpha) / 2$ and the domain is of the type:

$$
\frac{2(1+\eta) n \sigma\left(S^{d-1}\right)}{2-\alpha}\left(1-\left(\frac{2-\alpha}{2 n \alpha}\right)^{2 / 3}\right)<x^{\alpha}<\frac{3 n \sigma\left(S^{d-1}\right)}{2-\alpha} .
$$

Finally, observe that for $\alpha$ close enough to 2 or for $n$ large enough, $\varepsilon$ can be chosen arbitrarily small in (41). We thus recover a result as in [4] for $\alpha$ close enough to 2 .

$\mathrm{R}$ e $\mathrm{m}$ a r k 10. 1. The concentration inequalities (30) and (42) obtained in Sections 2.2 and 2.3 are of the same type. The ranges in (31) and (43) cannot however be completely compared, since we cannot compare $u_{1}(n, \alpha)$ and $1-((2-\alpha) /(2 n \alpha))^{2 / 3}$; this requires to study the sign of $h_{n, \alpha}\left(1-((2-\alpha) /(2 n \alpha))^{2 / 3}\right)$. In the limiting cases $\alpha \rightarrow 2$ or $n \rightarrow+\infty$, the ranges obtained is Section 2.2 are better.

2. Three types of concentration for the Lipschitz function of stable vector $f(X)$ are thus available. Roughly speaking, the regimes and their corresponding ranges are the following:

(a) for $x$ small, the order is $\exp \left(-c x^{\alpha /(\alpha-1)}\right)$;

(b) for $x^{\alpha}$ of order $\sigma\left(S^{d-1}\right)$, the order is $e^{-c x^{\alpha}}$;

(c) for $x^{\alpha}$ of order larger than $\sigma\left(S^{d-1}\right)$, the order is $c / x^{\alpha}$.

Here $c$ stands for a generic constant, different at each occurrence and which depends on $\alpha$ and on the dimension $d$.

\section{Poisson space with a stable Lévy measure of index $\alpha \in(0,2)$}

In this last section, we study $\mathbf{P}\{F-m(F) \geqslant x\}$, where $F$ is a stochastic functional on the Poisson space $\Omega^{\mathbf{R}^{d}}$ on $\mathbf{R}^{d}$ equipped with the stable Lévy measure $\nu$ given by (2). We recall that $\Omega^{\mathbf{R}^{d}}$ denote the set of Radon measures

$$
\Omega^{\mathbf{R}^{d}}=\left\{\omega=\sum_{i=1}^{N} \epsilon_{t_{i}}:\left(t_{i}\right)_{i=1}^{N} \subset \mathbf{R}^{d}, t_{i} \neq t_{j}, \forall i \neq j, N \in \mathbb{N} \cup\{\infty\}\right\},
$$

where $\epsilon_{t}$ is the Dirac measure at $t \in \mathbf{R}^{d}$. In the sequel, $\mathbf{P}$ is the Poisson measure with intensity $\nu$ on $\Omega^{\mathbf{R}^{d}}$. On the Poisson space, we have the linear, closable, finite difference operator

$$
D: L^{2}\left(\Omega^{\mathbf{R}^{d}}, \mathbf{P}\right) \longrightarrow L^{2}\left(\Omega^{\mathbf{R}^{d}} \times \mathbf{R}^{d}, \mathbf{P} \otimes \nu\right)
$$


defined via

$$
D F(\omega, x)=D_{x} F(\omega)=F(\omega \cup\{x\})-F(\omega) \quad d \mathbf{P} \times \nu(d \omega, d x) \text {-a.e., }
$$

where as a convention we identify $\omega \in \Omega^{\mathbf{R}^{d}}$ with its support, cf., e.g., [1] and the references therein.

In this section, we generalize the concentration bounds obtained in Section 2 to the case of stochastic functionals satisfying

$$
\left|D_{y} F(\omega)\right| \leqslant\|y\| \quad \mathbf{P}(d \omega) \otimes \nu(d y) \text {-a.e. }
$$

Roughly speaking, this hypothesis on $D F$ is analogous to $f$ being Lipschitz in Section 2. Since the mean may not exist anymore, concentration is expressed henceforth with respect to a median $m(F)$ of the stochastic functional $F$. Note finally that the case of Lipschitz function of stable vector $f(X)$ can be recovered, as a particular case, in this framework for any index $\alpha \in(0,2)$.

As explained in the introduction, configurations are truncated and we deal with the functional restricted to the truncated configuration $\omega_{R}=\omega \cap$ $B(0, R)$ with Lemma 2 below, while the rest of the configuration $\omega_{R}^{c}=$ $\omega \cap B(0, R)^{c}$ is controlled by some function $\gamma$. Wore precisely, we have

$$
\begin{aligned}
\mathbf{P}\{F-m(F) \geqslant x\}= & \mathbf{P}\left\{F-m(F) \geqslant x, \omega_{R}^{c}=\varnothing\right\} \\
& +\mathbf{P}\left\{F-m(F) \geqslant x, \omega_{R}^{c} \neq \varnothing\right\} \\
\leqslant & \mathbf{P}\left\{F_{R}-m(F) \geqslant x\right\}+\mathbf{P}\left\{\omega \in \Omega^{X}: \omega_{R}^{c} \neq \varnothing\right\} .
\end{aligned}
$$

The second summand on the right-hand side of (49) is dominated as in (6.2) in [1]:

$$
\mathbf{P}\left\{\omega \in \Omega^{X}: \omega \cap B_{X}(0, R)^{c} \neq \varnothing\right\} \leqslant \gamma(R),
$$

where $\gamma(R)$ can be chosen to be either $\gamma(R)=1-\exp \left(-\sigma\left(S^{d-1}\right) /\left(\alpha R^{\alpha}\right)\right)$ or $\gamma(R)=\sigma\left(S^{d-1}\right) /\left(\alpha R^{\alpha}\right)$. This choice will be discussed later.

Using the notation of the proof of Theorem 5.2 in [1], we have

$$
D_{y} g\left(F_{R}\right)(\omega) \leqslant\left|D_{y} F\left(\omega_{R}\right)\right| \leqslant|y|_{X} \quad \mathbf{P}(d \omega) \otimes \nu(d y) \text {-a.e., }
$$

where $g(x)=\left(x-m\left(F_{R}\right)\right)^{+} \wedge r$. Thus

$$
\sup _{y \in B_{X}(0, R)} D_{y} g\left(F_{R}\right) \leqslant R \quad \text { P-a.s. }
$$

and for $k \geqslant 2$

$$
\left\|D g\left(F_{R}\right)\right\|_{L^{\infty}\left(\Omega^{x}, L^{k}\left(\nu_{R}\right)\right)}^{k} \leqslant \frac{\sigma\left(S^{d-1}\right)}{k-\alpha} R^{k-\alpha} .
$$

First, we have as in (5.6) of [1]

$$
\mathbf{P}\left\{F_{R}-m\left(F_{R}\right) \geqslant y\right\} \leqslant \mathbf{P}\left\{g\left(F_{R}\right) \geqslant y\right\} \leqslant \mathbf{P}\left\{g\left(F_{R}\right)-\mathbf{E}\left[g\left(F_{R}\right)\right] \geqslant \frac{y}{2}\right\} .
$$


We next apply the following lemma to $g\left(F_{R}\right)$. This lemma deals with functionals on truncated configurations $F_{R}(\omega)=F\left(\omega_{R}\right)$ and is the counterpart of Lemma 1 in the same way as Lemma 5.5 in [1] is that of Lemma 2 in [3].

Lemma 2. Let $R>0$, and let $F: \Omega^{\mathbf{R}^{d}} \longrightarrow \mathbf{R}$ be such that

$$
\sup _{y \in B(0, R)}\left|D_{y} F(\omega)\right| \leqslant R \quad \mathbf{P}(d \omega) \text {-a.s. }
$$

For any $n \geqslant 2$, let $u_{n}(\alpha)$ be a unique nonzero (thus positive) solution of

$$
e^{u}-1-\frac{n-1}{2-\alpha} u=0
$$

and let

$$
u_{n}^{*}(\alpha)=\min _{1<k<(n-1+\alpha) / 2}\left(\left(k ! \frac{(n-1)(k+1-\alpha)}{(n-k)(2-\alpha)}\right)^{1 /(k-1)}\right) \wedge u_{n}(\alpha) .
$$

Then for all

$$
x \leqslant x_{0}:=n \frac{\sigma\left(S^{d-1}\right) R^{1-\alpha}}{2-\alpha} u_{n}^{*}(\alpha)
$$

we have

$$
\mathbf{P}\left\{F_{R}-\mathbf{E}\left[F_{R}\right] \geqslant x\right\} \leqslant \exp \left(-\frac{(2-\alpha) x^{2}}{2 n \sigma\left(S^{d-1}\right) R^{2-\alpha}}\right) .
$$

P r o of. The proof follows the lines of proof of Lemma 1 taking also into account the following changes. Starting from Proposition 2.2 in [1] in place of Theorem 1 in [2] we have (9) with the function $h_{R}$ given by

$$
h_{R}(s)=\sup _{\left(\omega, \omega^{\prime}\right) \in \Omega^{x} \times \Omega^{x}}\left|\int_{X}\left(e^{s D_{y} F(\omega)}-1\right) D_{y} F\left(\omega^{\prime}\right) \nu_{R}(d y)\right|, \quad s>0,
$$

where $\nu_{R}$ is the truncation of the measure $\nu$ on $B(0, R)$. Using the bounds $\left|D_{y} F\right| \leqslant R:=K$ for $y \in B(0, R)$, and for any $n \geqslant 2$ inequality (10), we have

$$
\begin{aligned}
h_{R}(s) \leqslant & \sum_{k=1}^{n-1} \frac{s^{k}}{k !} \sup _{\omega, \omega^{\prime} \in \Omega^{x}} \int_{X}\left|D_{y} F(\omega)\right|^{k}\left|D_{y} F\left(\omega^{\prime}\right)\right| \nu_{R}(d y) \\
& +\frac{e^{s K}-\sum_{k=0}^{n-1} s^{k} K^{k} / k !}{K^{n}} \sup _{\omega, \omega^{\prime} \in \Omega^{x}} \int_{X}\left|D_{y} F(\omega)\right|^{n}\left|D_{y} F\left(\omega^{\prime}\right)\right| \nu_{R}(d y) .
\end{aligned}
$$

Thus, using the inequality $x y \leqslant x^{p} / p+y^{q} / q, p^{-1}+q^{-1}=1$ and $x, y \geqslant 0$, we have for $q=k+1$ and $p=(k+1) / k, 1 \leqslant k \leqslant n$ :

$$
\begin{aligned}
\sup _{\omega, \omega^{\prime} \in \Omega^{x}} & \int_{X}\left|D_{y} F(\omega)\right|^{k}\left|D_{y} F\left(\omega^{\prime}\right)\right| \nu_{R}(d y) \\
\leqslant & \frac{k}{k+1} \sup _{\omega, \omega^{\prime} \in \Omega^{x}} \int_{X}\left|D_{y} F(\omega)\right|^{k+1} \nu_{R}(d y) \\
& +\frac{1}{k+1} \sup _{\omega, \omega^{\prime} \in \Omega^{x}} \int_{X}\left|D_{y} F\left(\omega^{\prime}\right)\right|^{k+1} \nu_{R}(d y)=\alpha_{k+1},
\end{aligned}
$$


where for all $k \geqslant 2$,

$$
\begin{aligned}
\alpha_{k} & =\|D F\|_{L^{\infty}\left(\Omega^{x}, L^{k}\left(\nu_{R}\right)\right)}^{k} \leqslant \int_{\left\{\|y\|_{2} \leqslant R\right\}}\|y\|_{2}^{k} \nu_{R}(d y) \\
& =\int_{S^{d-1}} \sigma(d \xi) \int_{\left\{\|r \xi\|_{2} \leqslant R\right\}} r^{k-1-\alpha} d r \leqslant \frac{\sigma\left(S^{d-1}\right)}{k-\alpha} R^{k-\alpha}:=\widetilde{\alpha}_{k} .
\end{aligned}
$$

Plugging this last bound into (52), we obtain an expression similar to (11) obtain in the proof of Lemma 1, we then finish the proof as in the end of the proof of Lemma 1 . Lemma 2 is proved.

Applying Lemma 2 for $g\left(F_{R}\right)$, i.e., for $y \leqslant 2 x_{0}=2 n\left(\sigma\left(S^{d-1}\right) R^{1-\alpha}\right) /$ $(2-\alpha)) u_{n}^{*}(\alpha)$, we get

$$
\begin{aligned}
\mathbf{P}\left\{F_{R}-m\left(F_{R}\right) \geqslant y\right\} & \leqslant \mathbf{P}\left\{g\left(F_{R}\right)-\mathbf{E}\left[g\left(F_{R}\right)\right] \geqslant \frac{y}{2}\right\} \\
& \leqslant \exp \left(-\frac{2-\alpha}{2 n \sigma\left(S^{d-1}\right) R^{2-\alpha}} y^{2}\right) .
\end{aligned}
$$

Now, with $y=R$,

$$
\mathbf{P}\left\{F_{R}-m\left(F_{R}\right) \geqslant R\right\} \leqslant \exp \left(-\frac{2-\alpha}{2 n \sigma\left(S^{d-1}\right)} R^{\alpha}\right),
$$

as long as, using (51),

$$
R^{\alpha} \leqslant \frac{2 n \sigma\left(S^{d-1}\right)}{2-\alpha} u_{n}^{*}(\alpha)
$$

In order to control $\mathbf{P}\left\{F_{R}-m(F) \geqslant x\right\}$, it is clear from (53), that we need to control $m\left(F_{R}\right)-m(F)$. To this end, we apply Lemma 5.1 in [1] with

$$
\begin{gathered}
\beta(R)=2 R, \quad \tilde{\gamma}(R)=\exp \left(-\frac{2-\alpha}{2 n \sigma\left(S^{d-1}\right)} R^{\alpha}\right), \\
R_{0}=\left(\frac{2 n \sigma\left(S^{d-1}\right)}{2-\alpha} u_{n}^{*}(\alpha)\right)^{1 / \alpha}
\end{gathered}
$$

and where the hypothesis $R \geqslant R_{0}$ in Lemma 5.1 of [1] is replaced by $R \leqslant R_{0}$, so that the final condition on $R$ is changed in the same way.

We thus have $m\left(F_{R}\right)-m(F) \leqslant R$, for all $R$ such that

$$
\inf _{0<\delta<1 / 2} \max \left(\gamma^{-1}(\delta), \widetilde{\gamma}^{-1}\left(\frac{1}{2}-\delta\right)\right) \leqslant R \leqslant\left(\frac{2 n \sigma\left(S^{d-1}\right)}{2-\alpha} u_{n}^{*}(\alpha)\right)^{1 / \alpha},
$$

where $\gamma(R)$ is given in (50). Hence, for $R=x / 2$

$$
\begin{aligned}
\mathbf{P}\left\{F_{R}-m(F) \geqslant x\right\} \leqslant \mathbf{P}\left\{F_{R}-m\left(F_{R}\right) \geqslant \frac{x}{2}\right\} & \leqslant \exp \left(-\frac{2-\alpha}{2 n \sigma\left(S^{d-1}\right)} R^{\alpha}\right) \\
& \leqslant \exp \left(-\frac{2-\alpha}{2 n \sigma\left(S^{d-1}\right)}\left(\frac{x}{2}\right)^{\alpha}\right) .
\end{aligned}
$$


From (49), it follows that

$$
\mathbf{P}\left\{F_{R}-m(F) \geqslant x\right\} \leqslant \exp \left(-\frac{2-\alpha}{2 n \sigma\left(S^{d-1}\right)}\left(\frac{x}{2}\right)^{\alpha}\right)+\gamma(x),
$$

as long as the condition (54) holds, i.e., in terms of $x$ as long as

$$
\inf _{0<\delta<1 / 2} \max \left(\gamma^{-1}(\delta), \widetilde{\gamma}^{-1}\left(\frac{1}{2}-\delta\right)\right) \leqslant \frac{x}{2} \leqslant\left(\frac{2 n \sigma\left(S^{d-1}\right)}{2-\alpha} u_{n}^{*}(\alpha)\right)^{1 / \alpha} .
$$

Next, we compare the two summands on the right-hand side of (55) using first $\gamma(R)=\sigma\left(S^{d-1}\right) /\left(\alpha R^{\alpha}\right)$ and next $\gamma(R)=1-\exp \left(-\sigma\left(S^{d-1}\right) /\left(\alpha R^{\alpha}\right)\right)$ in order to estimate the remainder term $\mathbf{P}\left\{\omega_{R}^{c} \neq 0\right\}$.

First choice for the function $\gamma$. In this part, taking $\gamma(R)=$ $\sigma\left(S^{d-1}\right) /\left(\alpha R^{\alpha}\right)$, we have the following theorem.

Theorem 4. Let $F$ be a stochastic functional on the Poisson space equipped with the $\alpha$-stable Lévy measure $(2), \alpha \in(0,2)$. Assume that

$$
\left|D_{y} F(\omega)\right| \leqslant\|y\| \quad \mathbf{P}(d \omega) \otimes \nu(d y) \text {-a.e. }
$$

Then for $\varepsilon$ satisfying (29),

$$
\mathbf{P}\{F-m(F) \geqslant x\} \leqslant(1+\varepsilon) \exp \left(-\frac{2-\alpha}{2 n \sigma\left(S^{d-1}\right)}\left(\frac{x}{2}\right)^{\alpha}\right),
$$

for all $x$ such that

$$
\begin{aligned}
& \left(\inf _{0<\delta<1 / 2} \max \left(\gamma^{-1}(\delta), \tilde{\gamma}^{-1}\left(\frac{1}{2}-\delta\right)\right)^{\alpha}\right) \vee \frac{2 n \sigma\left(S^{d-1}\right)}{2-\alpha} u_{1}(n, \alpha) \\
& \leqslant\left(\frac{x}{2}\right)^{\alpha} \leqslant \frac{2 n \sigma\left(S^{d-1}\right)}{2-\alpha}\left(u_{2}(n, \alpha) \wedge u_{n}^{*}(\alpha)\right),
\end{aligned}
$$

where $u_{1}(n, \alpha), u_{1}(n, \alpha)$, and $u_{n}^{*}(\alpha)$ are as in Theorem 2 with $\delta=1$.

This result generalizes Proposition 6.3 in [1] in the same way as Theorems 2 and 3 generalize Theorem 2 in [4].

P r o o f. As in Section 2, we compare the two summands in (55) studying the function $h_{n, \alpha}$ in (36). With the notation of Section 2 and for $\varepsilon$ satisfying (29), we derive (57) for

$$
\frac{2 n \sigma\left(S^{d-1}\right)}{2-\alpha} u_{1}(n, \alpha)<\left(\frac{x}{2}\right)^{\alpha}<\frac{2 n \sigma\left(S^{d-1}\right)}{2-\alpha} u_{2}(n, \alpha),
$$

and still under the condition (56), i.e., under (58).

Comments on the range of concentration in (58). 1 . We have

$$
\gamma^{-1}(\delta)=\left(\frac{\sigma\left(S^{d-1}\right)}{\alpha \delta}\right)^{1 / \alpha}, \quad \tilde{\gamma}^{-1}(\delta)=\left(\frac{2 n \sigma\left(S^{d-1}\right)}{2-\alpha} \ln \left(\frac{1}{\delta}\right)\right)^{1 / \alpha} .
$$


Since there is a unique solution $\delta_{0}(n, \alpha) \in\left(0, \frac{1}{2}\right)$ of the equation

$$
\frac{2-\alpha}{2 n \alpha}=\delta \ln \left(\frac{1}{1 / 2-\delta}\right)
$$

we have $\inf _{0<\delta<1 / 2} \max \left(\gamma^{-1}(\delta), \tilde{\gamma}^{-1}\left(\frac{1}{2}-\delta\right)\right)^{\alpha}=\sigma\left(S^{d-1}\right) /\left(\alpha \delta_{0}(n, \alpha)\right)$.

2. Moreover, since the left-hand side of (59) goes to zero as $\alpha \rightarrow 2$ or as $n \rightarrow+\infty$, we have $\delta_{0}(n, \alpha) \ln \left(1 /\left(\frac{1}{2}-\delta_{0}(n, \alpha)\right)\right) \rightarrow 0$ and thus $\delta_{0}(n, \alpha) \rightarrow 0$. It is also easy to deduce an equivalent for $\delta_{0}(n, \alpha)$ as $\alpha \rightarrow 2$ or as $n \rightarrow+\infty$ : $\delta_{0}(n, \alpha) \sim(2-\alpha)(2 n \alpha \ln 2)^{-1}$ where, for $x \rightarrow x_{0}, f(x) \sim g(x)$ means that $\lim _{x \rightarrow x_{0}}(f(x) / g(x))=1$. We thus have for $\alpha \rightarrow 2$ or for $n \rightarrow+\infty$,

$$
\inf _{0<\delta<1 / 2} \max \left(\gamma^{-1}(\delta), \tilde{\gamma}^{-1}\left(\frac{1}{2}-\delta\right)\right)^{\alpha} \sim \frac{2 n \sigma\left(S^{d-1}\right) \ln 2}{2-\alpha} .
$$

3. We have seen that $u_{2}(n, \alpha)>\ln (2 n \alpha \varepsilon /(2-\alpha))>1$. The upper bound of the domain (58) in Theorem 4 can thus be taken to be

$$
\frac{2 n \sigma\left(S^{d-1}\right)}{2-\alpha}\left(\ln \left(\frac{2 n \alpha \varepsilon}{2-\alpha}\right) \wedge u_{n}^{*}(\alpha)\right) .
$$

Comments on the bounds in the domain (58). For $\alpha$ close enough to 2 or for $n$ large enough, the limits (40) have been established in Section 2.2. Similarly, for any $\eta>0$ and for $\alpha$ close enough to 2 or for $n$ large enough, the domain (58) reduces to

$$
\begin{aligned}
& (1+\eta) \sigma\left(S^{d-1}\right)\left(\frac{2 n \ln 2}{2-\alpha} \vee \frac{\alpha}{\varepsilon}\right)<x^{\alpha} \\
& \quad<\left\{\begin{array}{lll}
\frac{2 n \sigma\left(S^{d-1}\right)}{2-\alpha} \ln \left(\sqrt{\frac{n-1}{2-\alpha}}\right) & \text { if } & \alpha \rightarrow 2, \\
\frac{6 n \sigma\left(S^{d-1}\right)}{2-\alpha} & \text { if } & n \rightarrow+\infty .
\end{array}\right.
\end{aligned}
$$

$\mathrm{R}$ e m a r k 11. 1. Theorem 4 complements Theorem 6.1 in [1] exhibiting for any $\alpha \in(0,2)$ a concentration regime in $\exp (-(2-\alpha) /$ $\left.\left(2 n \sigma\left(S^{d-1}\right)\right)(x / 2)^{\alpha}\right)$ for $x$ in a finite range, while Theorem 6.1 of [1] gives a concentration regime in $1 / x^{\alpha}$ for $x$ large enough, of order at least

$$
\sigma\left(S^{d-1}\right) \frac{2}{2-\alpha} \ln \left(\frac{2}{2-\alpha}\right) \ln \left(\frac{2}{2-\alpha} \ln \left(\frac{2}{2-\alpha}\right)\right) .
$$

2. When $\alpha$ is close to 2 , we recover a range of the same type as in Theorem 6.3 in [1].

Second choice for the function $\gamma$. Here, as in Section 2.3, we estimate the remainder term $\mathbf{P}\left\{\omega_{R}^{c} \neq 0\right\}$ using directly (4) rather than via the bound (5). We obtain the following result which is probably less sharp, but is given with more explicit bounds. 
Theorem 5. Let $F$ be a stochastic functional on the Poisson space equipped with the $\alpha$-stable Lévy measure $(2), \alpha \in(0,2)$. Assume that

$$
\left|D_{y} F(\omega)\right| \leqslant\|y\| \quad \mathbf{P}(d \omega) \otimes \nu(d y) \text {-a.e. }
$$

Then for any $\varepsilon$ satisfying (29),

$$
\mathbf{P}\{F-m(F) \geqslant x\} \leqslant(1+\varepsilon) \exp \left(-\frac{2-\alpha}{2 n \sigma\left(S^{d-1}\right)}\left(\frac{x}{2}\right)^{\alpha}\right)
$$

for all $x$ such that

$$
\begin{aligned}
& \left(\inf _{0<\delta<1 / 2} \max \left(\gamma^{-1}(\delta), \tilde{\gamma}^{-1}\left(\frac{1}{2}-\delta\right)\right)^{\alpha}\right) \\
& \vee \frac{2 n \sigma\left(S^{d-1}\right)}{2-\alpha}\left(1-\left(\frac{2-\alpha}{2 n \alpha}\right)^{2 / 3}\right)<\left(\frac{x}{2}\right)^{\alpha} \\
& <\frac{2 n \sigma\left(S^{d-1}\right)}{2-\alpha}\left(\left(1+\left(\left(\frac{2-\alpha}{2 n \alpha}\right)^{2 / 3} \wedge 0.68\right)\right) \wedge \frac{u_{n}^{*}(\alpha)}{2}\right) .
\end{aligned}
$$

Taking limits in (63) does not yield anything, since $1 \pm((2-\alpha) /$ $(2 n \alpha))^{2 / 3} \rightarrow 1$ as $\alpha \rightarrow 2$ or as $n \rightarrow+\infty$.

P r o of. As in Section 2.3, we compare the two summands in (55) studying now the function $g_{n, \alpha}$ in (45). As in Section 2.3, we derive (62) for $x$ in the range

$$
\begin{aligned}
0 & <\frac{2 n \sigma\left(S^{d-1}\right)}{2-\alpha}\left(1-\left(\frac{2-\alpha}{2 n \alpha}\right)^{2 / 3}\right)<\left(\frac{x}{2}\right)^{\alpha} \\
& <\frac{2 n \sigma\left(S^{d-1}\right)}{2-\alpha}\left(1+\left(\left(\frac{2-\alpha}{2 n \alpha}\right)^{2 / 3} \wedge 0.68\right)\right)
\end{aligned}
$$

and still under the condition (56), recovering the domain (63).

Lower range for $\alpha>1$. Finally, note that it is simple to verify that the counterpart of Theorem 1 holds for functionals on the Poisson space, namely, the following theorem holds.

Theorem 6. Let $F$ be a stochastic functional on the Poisson space equipped with the stable Lévy measure (2) with index $\alpha \in(1,2)$. Assume that

$$
\left|D_{y} F(\omega)\right| \leqslant\|y\| \quad \mathbf{P}(d \omega) \otimes \nu(d y) \text {-a.e. }
$$

Then for all $n$ large enough and for all

$$
\begin{gathered}
\lambda \in\left(\frac{\sigma\left(S^{d-1}\right)}{\alpha-1}\left(1+\sqrt{\frac{2(\alpha-1)^{2} n \sigma\left(S^{d-1}\right)^{(2-\alpha) /(\alpha-1)}}{\alpha(2-\alpha)}}\right),\right. \\
\left.\frac{\sigma\left(S^{d-1}\right)}{\alpha-1}\left(1+\frac{\alpha-1}{2-\alpha} n u_{n}^{*}(\alpha)\right)\right)
\end{gathered}
$$


there exists $x_{1}(n, \alpha, \lambda)>0$ such that for all $0 \leqslant x \leqslant x_{1}(n, \alpha, \lambda)$,

$$
\begin{aligned}
& \mathbf{P}\{f(X)-\mathbf{E}[f(X)] \geqslant x\} \\
& \leqslant \exp \left(-\frac{2-\alpha}{2 n \sigma\left(S^{d-1}\right)^{1 /(\alpha-1)}}\left(\lambda-\frac{\sigma\left(S^{d-1}\right)}{\alpha-1}\right)^{2}\left(\frac{x}{\lambda}\right)^{\alpha /(\alpha-1)}\right) \\
& \quad+\frac{\sigma\left(S^{d-1}\right)}{\alpha}\left(\frac{x}{\lambda}\right)^{\alpha /(\alpha-1)} \leqslant 1 .
\end{aligned}
$$

The comments made in Remark 3 and Remark 5 continue to apply to the stochastic functional framework.

For $\alpha \leqslant 1$, the lower range concentration requires to investigate the convergence of medians of truncated functionals which, at this point, requires more work.

\section{REFERENCES}

1. Breton J.-C., Houdré C., Privault N. Dimension free and infinite variance tail estimates on Poisson space. - Acta Appl. Math, 2007, v. 95, № 3, p. 151-203.

2. Houdré C. Remarks on deviation inequalities for functions of infinitely divisible random vectors. - Ann. Probab., 2002, v. 30, № 3, p. 1223-1237.

3. Houdré C., Marchal P. On the concentration of measure phenomenon for stable and related random vectors. - Ann. Probab., 2004, v. 32, № 2, p. 1496-1508.

4. Marchal $P$. Measure concentration for stable laws with index close to 2. - Electron. Comm. Probab., 2005, v. 10, p. 29-35.

Поступила в редакцию

9.XII.2004

Исправленный вариант

9.I. 2006 